\title{
REFLEXIONES EN TORNO AL IMPACTO DE PROGRAMAS DE EMPLEO PARA JÓVENES EN ARGENTINA. UN ESTUDIO DE CASO
}

\author{
MARÍA LOURDES FARÍAS*
}

\begin{abstract}
RESUMEN
El trabajo que aquí se presenta es parte de una investigación que tuvo dos objetivos principales, por un lado analizar el proceso de formación de políticas de empleo para jóvenes desplegadas desde el Estado y, desde una mirada más subjetiva, analizar los principales impactos sociales y económicos en los jóvenes destinatarios de esas políticas. Para poder llevarlo a cabo la investigación nos aproximamos al objeto de estudio a partir de distintos objetivos secundarios que permitieron caracterizar los principales criterios considerados para el diseño de las políticas y observar la adecuación de esas políticas a los problemas vivenciados por los jóvenes e identificar cuáles fueron las modificaciones en la vida de los jóvenes a partir de su inserción en los proyectos.

El trabajo de campo se realizó en Villa Itatí, Partido de Quilmes, Provincia de Buenos Aires. Las políticas analizadas fueron el Programa Manos a la Obra y el Programa Incluir. Para llevar adelante esta investigación utilizamos una metodología cualitativa y participativa, ya que la misma nos permitió acceder a la comprensión del fenómeno de investigación.
\end{abstract}

PALABRAS CLAVE: POLÍTICAS DE EMPLEO, JÓVENES, IMPACTO EN LA VIDA COTIDIANA

* Docente e investigadora de la Universidad Nacional de La Plata (UNLP), La Plata, Argentina. Integrante del Programa de Investigación Movimientos Sociales y Condiciones de Vida (UNLP). Licenciada en trabajo social y magíster en ciencias sociales del trabajo. Becaria doctoral del CONICET. Correo electrónico: lourdesfarias_mpd@yahoo.com. 


\title{
REFLEXÕES SOBRE O IMPACTO DE PROGRAMAS DE EMPREGO PARA JOVENS NA ARGENTINA: UM ESTUDO DE CASO
}

\begin{abstract}
Resumo
Este trabalho é parte de uma pesquisa que teve dois objetivos principias: por um lado, analisar o processo de formação de políticas de emprego para jovens desempregados por parte do Estado e, a partir de um olhar mais subjetivo, analisar os principais impactos sociais e econômicos sobre os jovens destinatários destas políticas. Para realizar a pesquisa, aproximei-me do objeto de estudo a partir de distintos objetivos secundários que me permitiram caracterizar os principais critérios considerados para o desenho das políticas, observar a adequação destas políticas aos problemas vivenciados pelos jovens e identificar quais foram as modificações na vida dos jovens a partir de sua inserção nos projetos.

O trabalho de campo foi realizado na Villa Itatí, Partido de Quilmes, Província de Buenos Aires. As políticas analisadas foram o Programa Mãos à Obra e o Programa Incluir. Para realizar esta pesquisa, utilizei uma metodologia qualitativa e participativa, já que a mesma me permitiu compreender o fenômeno investigado.
\end{abstract}

PALAVRAS CHAVE: POLÍTICAS DE EMPREGO, JOVENS, IMPACTO NA VIDA COTIDIANA

\section{REFLECTIONS ON THE IMPACT OF YOUTH EMPLOYMENT PROGRAMS IN ARGENTINA: A CASE STUDY}

\begin{abstract}
The work presented here is part of an investigation that had two main objectives, firstly to analyze the formation of youth employment policies deployed by the state and secondly, from a more subjective view, to analyze the major social and economic impacts in young recipients of these policies. In order to carry out this study, the subject was approached from different secondary targets which allowed me to characterize the main criteria taken into account when designing policies and monitoring the adequacy of those policies to the problems experienced by young people and identify what were the changes in the lives of young people from their inclusion in the projects.

The field work was realized in Villa Itatí, Quilmes, Buenos Aires. The policies analyzed were «Hands on», and the program «Included». To carry out this research a qualitative methodology and participatory was used, as it allowed access to understanding the phenomenon of the investigation.
\end{abstract}

KEY WORDS: EMPLOYMENT POLICIES, YOUNG PEOPLE, IMPACT ON DAILY LIFE 


\section{LOS COMIENZOS DE LA INVESTIGACIÓN}

Está la belleza y están los humillados; por difícil que sea la empresa no quisiera serle infiel ni a los segundo ni a la primera.

AlberT CAMUS

A FINES DE LA DÉCADA del 90, comenzó a hacerse visible una problemática que, si bien ya existía, tomo notoriedad a partir de su enunciación en el ámbito académico y político: el incremento de la población juvenil pobre. ${ }^{1} \mathrm{El}$ aumento de la desigualdad social, la precarizacion de las condiciones de educación y empleo, la marginación de vastos sectores de la población fueron componentes dominantes de ese fin de siglo y del comienzo de este. En ese contexto, tal como lo señalan el informe de Desarrollo Humano (1996) y el informe de la CEPAL (1997), los adolescentes y los jóvenes constituyeron una de las principales víctimas de la transformación estructural y de la crisis del mundo del trabajo y del Estado asistencial en todo el mundo y en particular en América Latina; Salvia (2000) los denomina la «generación pérdida».

Finalizando mi carrera de grado, como trabajadora social, no fui ajena a esta preocupación y comencé a realizar una pasantía en una ONG que tenía como objetivo favorecer los procesos de inclusión social de los adolescentes con menores oportunidades intentando acrecentar la visibilidad local de esta problemática. Mi primer trabajo fue en Villa Itatí (Quilmes) y nunca más pude dejarla.

En el año 2001, con un grupo de jóvenes de la Villa hicimos un relevamiento, casi censal, de distintos aspectos vinculados a los mismos. Los datos indicaban que sobre el total de la población joven entre 13 y 19 años encuestada solo un 24\% tenía la primaria completa y del $76 \%$ que no la había concluido, el $12 \%$ ya había abandonado sus estudios. En relación al nivel secundario, ${ }^{2}$ sólo un $5 \%$ lo había concluido y casi el $50 \%$ de los jóvenes que estaban en edad de cursarlo lo

1 Según datos del INDEC (1999), en el último semestre de 1999, el 20\% de la población nacional se encontraba en la franja entre 15 y 24 años, el 44\% de esos jóvenes no asistía a ningún establecimiento educativo, de ellos el 36\% no superó el primario completo y el 21\% no estudia ni trabaja. Esta cifra alcanza hoy en día, según el INDEC (2009) al 35\% de la población juvenil.

2 En ese momento denominado Polimodal por la Ley Federal de Educación. 
habían abandonado. A su vez los datos indicaban que los jóvenes que no estudiaban, pertenecían en su mayoría a hogares cuyos jefes se encontraban desocupados. Admitían los jóvenes en un $70 \%$ que era posible trabajar y estudiar al mismo tiempo, sin embargo solo un $10 \%$ lo hacía. El lugar del estudio se encontraba subordinado al trabajo. El 93\% acordaba en que estudiar ayudaba a conseguir trabajo; el $74 \%$ opinaba afirmativamente que muchos jóvenes irían a la escuela si se enseñara un oficio y el $85 \%$ estaba de acuerdo en que para conseguir el trabajo que les gustaría tener era necesario estudiar. Esto daba cuenta de la importancia que estos jóvenes le daban a la escuela, pero solo para posicionarse mejor de cara al trabajo, pensando en un futuro inmediato más que mediato.

Por otro lado, los relatos de los jóvenes confirmaban la existencia de hechos de discriminación como una importante problemática. El 55\% de la población joven encuestada contestó afirmativamente a la pregunta si se sentían discriminados, y la mayoría de ellos señalaba a los adultos y a la escuela como agentes de esa discriminación. Otra problemática que irrumpía era el tema de la participación; participar, era encontrar espacios donde ser y sentirse reconocido. El reclamo de espacios de participación se hacía oír en boca de los jóvenes que señalaban en un $68 \%$ que participaba poco y nada en las decisiones del barrio. En relación a esto, el 90\% de los jóvenes afirmaba que es importante participar pero, contradictoriamente, solo el $10 \%$ lo hacía en alguna institución, preferentemente, en comedores comunitarios.

Uno de los datos más significativos era que los valores defendidos, en muchos casos, como ideales, contrastaba con la problemática creciente del desempleo, la falta de oportunidades de movilidad social, la pérdida en la calidad de vida y prestaciones de servicios sociales, a lo que se sumaba, el padecimiento por las situaciones de exclusión.

Es por eso que empezamos a estudiar el tema juvenil direccionando la investigación hacia la problemática de la inserción laboral. Se estudiaron, por un lado, dos políticas de empleo destinadas a los jóvenes desarrolladas por el Estado nacional y el de la provincia de Buenos Aires durante el período 2004-2006: Manos a la Obra e Incluir, ${ }^{3}$ y por otro lado, se analizó el impacto de esas políticas en los jóvenes que fueran beneficiarios en Villa Itatí de la localidad de Quilmes.

3 Si bien el Programa Incluir no fue diseñado como una política de empleo, las propuestas de los proyectos presentados marcaron esa direccionalidad. 


\section{LOS JÓVENES EN ARGENTINA}

A lo largo de las últimas décadas, Argentina experimentó importantes transformaciones sociales, culturales y económicas que han dejado un saldo negativo en la estructura social. Los índices de marginación y pobreza se dispararon como consecuencia del modelo de exclusión adoptado por el país. Como afirman Svampa y Pereyra (2004) una importante porción de la sociedad quedó apartada del sistema dejando como saldo un proceso de desintegración del tejido social. La carencia de oportunidades y la inequidad incrementada en la última década en la Argentina, provocó que numerosos sectores de la población se vieran imposibilitados de ejercer sus derechos sociales y con ello sus posibilidades de desarrollo, no solo individual sino también colectivamente.

La crisis dio lugar a una nueva estructura social cuyos rasgos sobresalientes son el desempleo, el alto grado de desigualdad en cuanto al acceso a los bienes y las nuevas formas de pobreza (Barbeito y Lo Vuolo, 1995). Se hace evidente que cada vez son más personas las que están debajo de la línea de pobreza y niveles de indigencia, la mayoría de las cuales se ubican en villas, asentamientos y barrios precarios del conurbano sin posibilidades de satisfacer sus necesidades básicas en relación a la vivienda, salud, educación, nutrición, etcétera.

En este marco, los jóvenes, y más aún los jóvenes en situación de pobreza, han sido y son uno de los sectores más perjudicados por la aguda crisis de la región. Las altas tasas de desocupación, que en promedio duplica la tasa de la población adulta junto con la baja calidad y escasa productividad del trabajo al que pueden acceder, se ha convertido en uno de los problemas públicos centrales (Jacinto, 2004; Miranda, 2007; Salvia, 2005).

Este grupo etario ha sufrido con especial rigor las consecuencias del desempleo y su inserción en el mercado laboral es precaria; estando en muchos casos imposibilitados de continuar con sus estudios o conseguir trabajo - ya sea formal o informal—, lo que reduce cada vez mas sus posibilidades de afiliación social (Castel, 1997). ${ }^{4}$ La for-

4 El autor señala que la idea de desafiliación implica también una disociación con los mecanismos tradicionales de integración social, provocados por la precarización laboral, y prefiere ese vocablo frente a la denominación genérica de exclusión social. 
mación con la que las nuevas generaciones se incorporan al mercado laboral es deficiente. La fragmentación social se construye desde los inicios de la vida laboral y el sistema educativo opera como un mecanismo de reproducción generacional de la pobreza, ya que por ejemplo entre los hogares de más bajos ingresos los jóvenes sufren repitencia muy tempranamente y, en la mayoría de los casos, concluye con la deserción antes de terminar la educación media.

Entre 2003 y 2007, el mercado laboral argentino presentó mejoras en la posibilidad de crear puestos de trabajo. Sin embargo, el nuevo modelo económico se enfrentó a una situación compleja debido a que ante una pequeña reactivación o intento de localización de una nueva industria no existía el personal técnico especializado (INDEC, 2004). ${ }^{5}$ Esta situación motivó a que el Estado promulgara en el año 2005 la Ley de Educación Técnica ( $\left.\mathrm{N}^{\circ} 26.058\right)$ con los objetivos de ordenar y regular la educación técnico profesional, mejorando y fortaleciendo sus instituciones y programas a través del apoyo técnico y financiero, y para creara con ese objetivo un Fondo Nacional para su financiamiento. Estas medidas tuvieron un rápido impacto ya que se observó un incremento entre 2004 y 2006 de un 15,26\% de la matrícula en las escuelas técnicas de la Provincia de Buenos Aires. En otras palabras, 15 mil alumnos se sumaron a este modelo educativo en los últimos años (DGCE, 2006). El balance de la Ley Federal de Educación promulgada en los noventa fue negativo para todo el sistema educativo (Salvia, 2000; Filmus, 2004). Es ilustrativo lo que señalaba en una entrevista una responsable de una organización comunitaria de la Villa Itatí al decir:

Muchos chicos tienen que salir a trabajar. Hay chicos muy chiquitos que dejan el colegio, se van a Constitución a abrir puertas (A).

En la Cava no hay matriculación en el nivel secundario, y en el primario no supera el $20 \%$ y entre los que van, muchos van por la comida que les da la escuela. Hay muchísima deserción escolar porque los adolescentes son el único sostén de familia, y por la dificultad de comunicación por los diferentes códigos culturales de la escuela y de los sectores marginales (A).

5 El Instituto Nacional de Estadísticas y Censos (INDEC) comenzó a publicar en 2004 el Índice de Demanda Laboral Insatisfecha. En el último trimestre de 2007, la demanda insatisfecha total ascendía al $45 \%$. De este porcentaje, el $30 \%$ corresponde a trabajadores calificados y el restante 15\%, a personal sin calificación. 
Paralelamente a los mecanismos que favorecen el incremento de la pobreza, se activan otros que aumentan el aislamiento juvenil respecto de los demás estratos sociales: i) la segregación residencial; ii) la separación de los espacios públicos de sociabilidad informal (fuera del mercado); iii) la segmentación de los servicios básicos, por ejemplo, la educación.

A raíz de este aislamiento social los jóvenes quedan marginados de otras influencias que pudieran brindarle algún camino para construir su identidad y sentido de pertenencia.

Según Touraine (2000) se requiere de un trabajo arduo que es abandonar el realismo ingenuo, que cree que de lo único que se trata es de descubrir los problemas reales de la juventud para elaborar luego una política en la que se dé una respuesta apropiada ya que sin trabajo, sin redes de contención, sin las habilitaciones educativas y sociales exigidas por el mercado, ni oportunidades para obtenerlas, los jóvenes más pobres quedan afuera de la sociedad formal y como sostiene Salvia (2000) «se refugian en las estructuras no visibles de la pobreza y la marginalidad. Finalmente, tanto el mercado como el orden social oficial los sospecha, los persigue y los juzga como delincuentes».

Por todo lo anteriormente mencionado es que sostenemos que el impacto de una política de empleo no puede circunscribirse al porcentaje de jóvenes que ingresaron al mercado laboral, sino que debe tener en cuenta las modificaciones en los distintos ámbitos de su vida personal y social.

\section{LOS JÓVENES DE VILLA ITATÍ}

La villa está ubicada en el sudoeste del Conurbano Bonaerense. Abarca un perímetro de 5 kilómetros, entre el Acceso Sudeste y las calles Montevideo, Levalle y Ayacucho. Según la Municipalidad de Quilmes esas tierras pertenecen a Vialidad Nacional y su cesión a los vecinos «es difícil de concretar a corto plazo». El mapa dibuja 36 manzanas asimétricas donde viven alrededor de 8.000 familias. El nombre de Itatí llegó junto con la devoción a la Virgen que trajeron los que llegaron del litoral, pero la historia de la villa empezó a escribirse con trazos del Paraguay. La memoria de los vecinos dice que los primeros en asentarse fueron las familias de Eliseo Núñez y Eleodoro Ríos, que llegaron a Argentina en 1961. La población aumentó al mismo ritmo que la desocupación, el cierre de fábricas en la zona y los ajustes económicos en nuestro país y en 
los vecinos. Todos llegaron en busca de horizontes que jamás alcanzaron y se acomodaron como pudieron. Los que tenían algún familiar en la villa, adosaron alguna pieza o agregaron otras en el techo. Lo que originalmente era un par de ambientes para toda una familia — con diez integrantes promedio- ahora se ensambla en dos pisos, más o menos encuadrados. Según el censo realizado en el 2002 por distintas organizaciones sociales en la villa viven alrededor de 60.000 personas de las cuáles aproximadamente el 50\% está comprendida en la franja de 13 a 19 años. La mayoría de sus habitantes son cartoneros que se dedican a la recolección de basura como única alternativa laboral, actividad conocida en la Argentina como cirujeo. Descargan lo que juntan a un costado del acceso y seleccionan la basura antes de ir a venderla como cartón, vidrio, plástico o lata. Algunos tienen un caballo para tirar del carro, pero hasta esto se convirtió en un lujo. Es un privilegio solo de algunos «o come el caballo o comemos nosotros», dicen los que andan a pie. Las casillas se levantan de un día para otro, en forma anárquica, en el espacio que esté libre. Se cuelgan de los cables de luz para alimentar heladeras viejas que apenas enfrían; no tienen redes de alcantarillados y mucho menos desagües sanitarios. Al borde del Acceso Sudeste ${ }^{6}$ la villa se hunde en un enorme foso conocido como «La Cava». Una depresión que ocupa el $20 \%$ de la superficie, hundida varios metros, que se formó cuando se extrajo la piedra caliza con la que se construyó gran parte del acceso inaugurado el 15 de diciembre de 1978. Para el resto de la villa, es «el fondo» o «allá abajo» y no son eufemismos: ahí viven cientos de personas en casillas de chapa, a orillas de una depresión inundada por pozos ciegos y las napas freáticas. El Municipio de Quilmes instaló un par de bombas de desagüe que durante todo el día saca agua putrefacta hacia un canal pluvial de la zona. Cada vez que llueve, como los terrenos están en declive y forman un pozo, la villa se hunde pero estas inundaciones se agravan cuando suben el nivel de las napas de agua. En la villa, ese sector es conocido como «la laguna». Y a veces hasta cumple esa función, ya que cuando el nivel sube lo suficiente, los más chicos se meten en carcasas de heladeras desarmadas y «navegan» sobre el agua estancada. «La Cava» es lacerante. La basura es lacerante. El hedor es lacerante. Las necesidades son lacerantes. Gran parte de la población joven está ociosa y en muchos casos posee conductas adictivas. De ningún modo pretendemos establecer correlaciones mecánicas entre pobre-

6 Vía de acceso rápido entre Buenos Aires y Quilmes, es un camino alternativo a la autopista Buenos Aires/La Plata. 
za, adicción y violencia, sino que consideramos que es necesario identificar a los sectores y situaciones de mayor vulnerabilidad que predisponen a la violencia, subrayando entre ellas la situación particular de los jóvenes, que como bien señala Rodríguez (2005) «enfrentan un agudo cuadro de exclusión económica, social, política y cultural, que los mantiene alejados de la dinámica central de nuestras sociedades y los obliga a refugiarse —en muchos casos- en los márgenes de nuestras ciudades y pueblos».

La ausencia de referentes familiares, la insuficiente contención institucional y el alto índice de deserción escolar son «moneda corriente» y condicionan fuertemente la perspectiva de futuro en los jóvenes. Por otra parte, la Villa tiene una larga historia de lucha para conseguir mejores condiciones de vida y han logrado concretar proyectos comunitarios basados, fundamentalmente, en su capacidad organizativa y movilizadora.

En diferentes entrevistas realizadas a los adultos de esas instituciones, nos expresaban lo que ellos entendían era la problemática de los jóvenes:

Están sin ninguna expectativa, sin ningún tipo de educación ni nada, sin ningún recurso, solamente Poxiran para matar el hambre (E).

La droga es la excusa para el diálogo entre amigos, así como en otros grupos sociales puede ser tomar un café. Los pibes suelen decir «yo paro con tal grupo» —esa es su presentación, su identidad-, y no les importa si ese grupo es el que tiene la fama de ser el más violento o el que más roba, a algunos, hasta les da orgullo $(\mathrm{N})$.

Decimos que hay falta de educación sexual, porque cada vez hay más embarazo en adolescentes (E).

Además del embarazo que es un problema en sí, cómo lo van a educar, cómo lo van a mantener... está el problema del SIDA (V).

Para los adultos además, la falta de trabajo que deja sin oportunidades a los jóvenes es visualizada como la fuente principal de muchos conflictos evitables. Los jóvenes son presionados o forzados a aceptar trabajos insalubres, como el cirujeo, la venta de droga y/o de pegamento con tolueno, o de mercadería robada, u otras actividades producto de la exclusión total. La posibilidad de inserción laboral es nula por pertenecer a la villa, por no haber finalizado el secundario, por no tener preparación técnico-profesional. Destacaron asimismo la creciente desintegración familiar y la incapacidad de las instituciones para contener a esta población. 
La propia familia desintegrada que prácticamente expulsa el chico a la calle. En la falta de contención yo quiero agregar que no solamente la familia, las instituciones que estamos trabajando con estos chicos nos desbordan las situaciones. Entonces nos desborda a la familia, nos desborda a nosotros que estamos todos los días con ellos, a las instituciones y también a los profesionales y especialistas que están trabajando con ellos. Es más amplia la falta de contención (N).

Es común verlos en diferentes esquinas, como su lugar de socialización y de contención; buscan ser alguien a través de ese grupo donde la droga puede funcionar como un rito de iniciación.

\section{ALgUNOS IMPACTO DE LOS PROGRAMAS EN LOS JÓVENES}

Los jóvenes focalizados en estas políticas fueron aquellos cuya situación de vida era más crítica, cuya situación de vulnerabilidad psicoso$\mathrm{cial}^{8}$ les impida desarrollar su proyecto vital. Siguiendo a Boari (2002) nos referimos a jóvenes que sin contar con una referencia familiar contenedora que posibilite procesos de identificación positiva, están en un franco proceso de desafiliación de espacios de desarrollo humano integral. No están incluidos en espacios laborales ni de educación formal ni de capacitación en oficios, no participan en grupos u organizaciones juveniles o en organizaciones comunitarias y que hayan tenido episodios de conflicto con la ley o bien estén en peligro de tenerlos.

Nos parece importante desagregar cada una de estas variables presentes en los jóvenes que no son contempladas al momento de diseñar e implementar un plan, programa o política pública.

\section{a) No inclusión en espacios de educación formal}

La situación de crisis económica que atraviesan las familias de estos jóvenes — padres en situación de subocupación o desocupación-, configura muchas veces la necesidad de que todos los miembros de la familia, incluyendo niños y adolescentes, deban aportar económicamente a la supervivencia familiar. Si a esto le sumamos que la educación no es visualizada por estos sectores sociales como espacios capaces de mejo-

8 Definida la vulnerabilidad psicosocial como aquel daño psíquico que ha sufrido un sujeto por no tener acceso a la satisfacción de sus derechos humanos fundamentales como: salud, educación, alimentación, seguridad, trabajo, etcétera. 
rar la situación de vida y provocar movilidad social, se entiende la razón de la priorización por parte de estas familias, de la generación de ingresos por parte de sus hijos sobre la actividad escolar. Estos jóvenes sostienen una presencia irregular en la escuela que, por otra parte, mantiene una escasa adecuación a sus necesidades y experiencias. Muchas veces, además, no cuentan con elementos básicos, como ropa, útiles, etcétera, necesarios para la concurrencia escolar. A partir de éstas y otras situaciones, los jóvenes se alejan progresivamente de los espacios de educación formal perdiendo — sobre todo— al llegar a la adolescencia la vinculación con la organización escolar.

\section{b) No inclusión en espacios de educación en oficios}

La inclusión en espacios de capacitación en oficios, si bien podría constituirse en una respuesta acorde a las posibilidades de estos jóvenes, se ve dificultada en razón de que muchas veces desconocen su existencia o no la consideran atrayente dado que no coinciden con las expectativas de movilidad social fantaseada. El abandono a la concurrencia de estas capacitaciones informales en oficios se debe, entre otras cosas, a una inadecuación de estas ofertas de capacitación laboral a la situación de estos jóvenes, ya que por ejemplo, no considera aspectos socioeducativos del entorno familiar, de los marcos culturales, de las situaciones migratorias de gran parte de ellos.

\section{c) No inclusión en espacios laborales}

No sólo hay que tener en cuenta la escasa demanda laboral actualmente existente en el mercado sino también el hecho de que estos jóvenes no cuentan con estudios, capacitación, experiencia laboral previa y muestran, muchas veces, deficiencia en habilidades funcionales básicas, elementos todos que conforman una situación de desventaja en cuanto a oportunidades laborales.

Por otro lado, según Pichón Riviere (1978), la desestructuración del tiempo y del espacio propio de la adolescencia así como su desarrollo dentro de modelos laborales familiares caracterizados por la inestabilidad y la irregularidad en sus tiempos, conforma un cuadro caracterizado por la dificultad en el sostenimiento de encuadres estables. 


\section{d) No inclusión en organizaciones comunitarias}

Los jóvenes no perciben que las organizaciones comunitarias sean espacios de participación para ellos. Consideramos que esto se debe en parte a que la desconfianza en las instituciones, en general, así como la cooptación y las prácticas relacionadas al clientelismo político que se desarrollan, a veces, a través de las organizaciones comunitarias ayuda - muchas veces - a que los jóvenes posean una escasa confianza y referencialidad respecto de las organizaciones barriales. Por otro lado, en la mayoría de los casos, no existen espacios para jóvenes en esas organizaciones comunitarias. Tampoco se presenta, a partir de las mismas, propuestas atractivas que les permitan incluirse a partir de sus intereses. También faltan en las organizaciones comunitarias líderes socioeducativos capacitados para trabajar con jóvenes.

\section{e) No inclusión en organizaciones juveniles}

Los jóvenes a los que nos referimos no son parte de las organizaciones juveniles, ya sea porque no existe este tipo de organización, o bien, porque no visualizan la posibilidad de hacerlo. Esta falta de motivación puede deberse, entre otras cosas, a un desconocimiento de las posibilidades que le ofrece esta participación, la falta de modelos en esa dirección y la escasa existencia de líderes juveniles capaces de movilizar hacia procesos de inclusión positiva. Por otro lado, la despolitización y fragmentación de la sociedad genera una situación en la que los jóvenes no se ven como actores sociales con intereses sectoriales propios, y con la capacidad de provocar cambios en sí mismos y los demás manteniendo así un proceso de adaptación activa a la realidad.

\section{f) Autoimagen negativa}

La vivencia de fracaso en sus procesos de inclusión social, el sentimiento de segregación y exclusión, la dificultad para la realización de procesos de identificación positiva con otros jóvenes, el exceso de tiempo libre improductivo se une muchas veces a una historia familiar e institucional que no los ha provisto de procesos de socialización adecuados, con escasa posibilidad de diálogo y simbolización de sus experiencias desencadenando, en algunos, procesos de encapsulamiento personal y desarrollo de un sistema defensivo, desobjetivación y despersonalización donde lo simbólico —que nos instituye como suje- 
tos de la cultura - se precariza, y se produce un desdibujamiento de las normas de convivencia social, en tanto que al no percibirse a sí mismos como sujetos de derecho no pueden visualizar al otro dándose situaciones de acting out. ${ }^{9}$

La relación con jóvenes que en su misma situación han entrado en conflicto con la ley y la identificación con estos líderes, la dificultad para evaluar realísticamente la relación medios y fines propios de la adolescencia y la incidencia negativa de los medios de comunicación con la exacerbación del consumo, el facilismo y los parámetros de éxito conforma un cuadro donde la resolución de sus necesidades a partir del delito puede comenzar a constituir una posibilidad cierta por lo que se hace imperiosa la necesidad de generar políticas públicas que los incluyan como principales beneficiarios.

Los objetivos de ambos programas estudiados — Manos a la Obra e Incluir - se propusieron generar para los jóvenes en situación de pobreza mayores oportunidades de inserción económica y social proporcionándoles capacitación en un oficio a nivel de semicalificación, formación personal, la posibilidad de iniciarse en el autoempleo y, en algunos casos, brindarles alguna experiencia laboral. Su inspiración radicaba en que para el gobierno nacional era central la búsqueda de mecanismos que permitieran lograr un crecimiento económico sostenible con la equidad necesaria para los sectores de más escasos recursos en donde los jóvenes pudieran aportar al ingreso familiar y ayudaran a generar redes sociales en la comunidad de referencia.

El cumplimiento de este compromiso se manifestaba en la implementación de las medidas apropiadas que ayudaran a la población objetivo lograr una mejor preparación para el mundo del trabajo. Estas acciones debían ser un estímulo al desarrollo de la iniciativa personal, al fortalecimiento de su entorno social, al acceso a los beneficios del desarrollo del país y, finalmente, al mejoramiento de la calidad de vida y del entorno familiar como una fórmula necesaria para romper el círculo de la pobreza.

9 «Acciones que presentan casi siempre un carácter impulsivo relativamente aislable en el curso de sus actividades, en contraste relativo con los sistemas de motivación habituales del individuo, y que adoptan a menudo una forma auto o heteroagresiva. Es una demanda de simbolización exigida en una transferencia salvaje» (Chemama, 1996:2). 
Estos principios validaban la necesidad de ampliar el horizonte, de percibir la capacitación no sólo como la realización de cursos que permitieran la adaptación de los jóvenes al sistema productivo sino que debía entenderse como una herramienta para incentivar la inserción social y laboral efectiva de jóvenes de ambos sexos. Por lo tanto, los programas debían ser concebidos como una acción transitoria de un fenómeno social de envergadura como era la situación de desintegración de los jóvenes y las tasas de desempleo que los afectaban.

Otros de los aspectos considerados, en los diagnósticos previos al diseño e implementación de los programas, ${ }^{10}$ fue el análisis de las fortalezas y debilidades del sistema de educación y la identificación de las capacidades de éste para dar respuesta adecuada a los problemas de la población objetivo. La enseñanza que arrojaba la implementación del programa estaba dirigida a que este tipo de acciones debían ir acompañadas, en forma simultánea, con medidas tendientes a producir modificaciones en el sistema educativo nacional buscando un vínculo más estrecho entre educación y trabajo.

Otro de los aspectos considerados, explicaba un funcionario nacional en el lanzamiento del Incluir, era que «la cobertura del programa debía ser acorde con la generación de puestos de trabajo y el crecimiento económico del país, de lo contrario se crearían aspiraciones desmedidas, introduciendo distorsiones dañinas al mercado laboral y podría generar a la larga, un conflicto social, particularmente en aquellos jóvenes que habían asumido el compromiso de superar su condición, con la esperanza de que la capacitación recibida fuera el instrumento adecuado para superar su situación de desintegración social».

Podemos identificar como puntos en común entre ambos proyectos, que ambos pusieron más énfasis en la búsqueda de una integración de tipo social y cultural más cercana a la noción de ampliación de ciudadanía que a una búsqueda efectiva de inserción laboral. Acorde con ello, las organizaciones ejecutoras promovieron la transferencia y apropiación de conocimientos en temáticas transversales (liderazgo juvenil, voluntariado, participación comunitaria, derechos juveniles, promoción de la salud, etcétera) y la implementación de actividades culturales (teatro, video, artes plásticas, deportivas y recreativas, etcétera), intentando promover la integración de los jóvenes al sistema social.

Este sesgo de los proyectos derivó en una falta de énfasis en cuáles eran las modificaciones esperadas en relación al abordaje específico que

10 www.desarrollosocial.gov.ar. 
debía considerarse frente al problema del empleo juvenil. Una de las principales dificultades fue que el diseño —si bien previó instancias de financiamiento para la concreción de proyectos productivos-, se centró básicamente en acciones de intervención a través de la formación. Esto surge de la suposición de que el joven no consigue trabajo por carecer de preparación. Si bien, cuando se enumeran los objetivos de los programas, se señalan otras causas más específicas, como la insuficiente información que los jóvenes poseen sobre el mercado del trabajo local, la carencia de herramientas para realizar un diagnóstico certero de las necesidades productivas locales o la falta de habilidades autogestivas para la concreción del autoempleo, el problema sigue estando definido, principalmente, como una carencia formativa.

Como sostiene Barbetti (2007:11) «este argumento que une causalmente el desempleo con la falta de formación, que ha sido sumamente cuestionado y continúa siendo objeto de un profundo debate, se vincula con el renovado auge de las teorías económicas que - desde ideas productivistas como excelencia, calidad y eficiencia - tratan de afirmar la existencia de una relación lineal entre educación y empleo. Concretamente desde la teoría del capital humano — principal soporte teórico de las políticas públicas de empleo juvenil en los 90 y que aún se mantiene vigencia - se sostiene que la formación es un acervo de conocimientos y habilidades que se convierte, gracias al apoyo del Estado, en una fuente de recursos de la que pueden disponer los ciudadanos. El que más capital de este tipo acumula antes de ir al mercado de trabajo, o ya estando en él, más opciones tiene para obtener un empleo adecuado a sus intereses o expectativas y para desarrollar una carrera profesional».

Esta concepción teórica, considera a la formación de los individuos como la herramienta que permite igualar oportunidades, ya que se asume que el mercado laboral es homogéneo, sin barreras y que de acuerdo a sus capacidades los individuos pueden realizar un libre intercambio entre diferentes puestos sociales (Cachón, 1989).

A lo largo de nuestra investigación observamos que el Estado no es eficaz a la hora de lograr los objetivos propuestos por los programas. ${ }^{11}$ De los tres microemprendimientos realizados —panadería, electricidad y diseño de página web- ninguno terminó siendo una

11 Entre sus objetivos está la formación para la obtención de un empleo asalariado, la posibilidad de que los jóvenes puedan una vez finalizada la capacitación generar un emprendimiento propio, sustentable, orientado a las demandas locales y con un carácter asociativo. 
experiencia «exitosa» ya que no se sostuvieron en el tiempo. Tal como lo define Jaramillo (2004), estas políticas de gobierno apuestan «por el poder de uno»; es decir, parten del supuesto de que cada joven tiene el poder de crear un autoempleo por sus propios medios. En el caso de los jóvenes, lo que se intentó promover desde el programa fueron las ideas vinculadas con el diagnóstico social participativo, el asociativismo y el cooperativismo como instrumentos útiles para la generación y puesta en marcha de proyectos productivos así como el reconocimiento de los beneficios que supone la generación de un sistema de redes sociales en la búsqueda de su inserción laboral.

Como plantea Barbetti (2007:14) «de esta manera, el enfoque del emprendimiento y del 'sujeto emprendedor', —mirada comprensiva construida principalmente desde la teoría económica y ligada al mundo de los negocios-, aparece extrapolada también a la esfera de lo social y se cristaliza en apuestas concretas que le atribuyen el estatus de mecanismo de inserción laboral en el terreno de las políticas y programas de empleabilidad asociándolas, como en el caso analizado, con la perspectiva del capital social». ${ }^{12}$

También es importante señalar que ninguno de los programas analizados concibió en su diseño inicial líneas de capacitación con componentes diversificados en especialidades diversas que consideraran las especificidades y características propias de los diversos grupos etarios; es decir, el mayor o menor grado de inserción social, riesgo psicosocial y el mayor o menor nivel de experiencia en el campo laboral.

La implementación realizada en Villa Itatí fue a partir de organizaciones no gubernamentales quienes plantearon estrategias de intervención similares en ambos proyectos que incluyeron capacitación y puesta en marcha de microemprendimientos. La duración de los proyectos fue de dieciocho meses.

No obstante, a partir de las entrevistas realizadas a los responsables y técnicos encargados de su aplicación se desprende que la apuesta era diseñar estrategias de largo plazo en el abordaje de la participación de los jóvenes y su inserción en el mundo del trabajo de manera digna y calificada. Asimismo, desde las organizaciones se concibe la reforma educacional implementada en el 2001, como un elemento que

12 Se entiende por capital social la definición dada por la CEPAL (2001) al denominar a esto al conjunto de normas, instituciones y organizaciones que promueven la confianza y la cooperación entre las personas, las comunidades y la sociedad en su conjunto. 
contribuyó a empeorar las condiciones laborales futuras de muchos jóvenes en los barrios. La responsable de la organización Intihuasi declara en una entrevista: «La implementación del EGB 1, 2 y 3 y del polimodal eliminaron la oferta de formación en oficio. Las escuelas industriales cierran, y esta reforma educacional hace que los jóvenes a los 15 años no tengan la posibilidad de aprender algún oficio (electricidad, carpintería, tornería, etcétera). Por eso se vuelve cada vez más difícil encontrar un empleo bueno para los que no tienen la posibilidad de seguir estudiando».

A la falta de oportunidades hay que sumar la historia familiar de la mayoría de los jóvenes con quienes trabajaron las organizaciones del proyecto, en muchos casos, se trata de familias sin una historia laboral permanente en el mercado formal ni en el informal; los padres de estos jóvenes dependen, casi exclusivamente, de los subsidios estatales de los distintos planes sociales. Éstos son los únicos ingresos seguros. Los lugares de trabajo se transforman, para ellos, en algo lejano, sin sentido. Al referirse a ello algunos técnicos del programa expresaban:

Familias de cinco hijos que de pronto no pueden pagarle el boleto para ir a la escuela a los cinco, y se quedaba afuera el más grande, el responsable... no sé qué causó más que los pibes se fueran de la escuela: si la necesidad de conseguir plata o de no tenerla para ir (Mujer, 35 años).

Si bien se puede afirmar que es posible que el Estado realice una política activa en materia de capacitación de carácter masivo, los resultados obtenidos no reflejan eficiencia y un alto impacto. En el caso particular de la Villa Itatí ambos programas tuvieron una cobertura que no superaba el 3\% de la población joven en situación de pobreza.

La adecuada focalización de los beneficiarios es un aspecto relevante de los Programas si se pretende impactar en la población objetivo. Ambos programas apelaron a la «autofocalización» como criterio de incorporación, de los beneficiarios. Esto es así porque el Estado se retiró de la ejecución directa y apeló a la participación de terceros como organismos no gubernamentales en su mayoría y, en algunos casos, al municipio a través de la dirección de juventud, a quienes corresponde establecer estrategias de difusión para la incorporación de los jóvenes.

Sin embargo hay dos situaciones diferentes en la descripción de los beneficiarios en los proyectos. En el caso del Incluir al ser un proyecto específicamente destinado a los jóvenes, los beneficiarios estaban pre- 
viamente identificados: debían tener entre 18 y 25 años, alojarse, preferentemente, en zonas urbanas, en condiciones de exclusión como pobreza, desescolarización y falta de oportunidades educativas. Estas condiciones, los marginaban de los ámbitos laborales o los situaban en el mercado informal y/o en el manejo de prácticas ilegales. Era intención del programa cambiar radicalmente la situación de estos jóvenes.

La lógica del PMO fue diferente ya que al no estar diseñado para los jóvenes, la selección dependió de los criterios de quienes lo implementaron. Vale aclarar que por ser un programa tendiente a la inserción laboral a través de emprendimientos productivos los jóvenes debían ser mayores de 18 años. Para las organizaciones, esto generó algunas dificultades de implementación ya que no estaban previamente anticipadas estas líneas de corte y muchos, jóvenes menores de esa edad, habían empezado a participar del programa, pero posteriormente no pudieron recibir ningún beneficio. Esto generó y genera, no solo con este programa, una situación de desaliento en los jóvenes que perciben que nunca llega su turno o desconfían de las promesas de los adultos.

Como señalara anteriormente queda claro, a partir de las entrevistas a los profesionales que han trabajado en ambos proyectos, que la intervención, desde el principio, tenía la intención de ir más allá de la contención de problemas puntuales o de la entrega de asistencia inmediata. Por sus historias de vida, por el lugar donde les tocó nacer y vivir, por la situación del país, los jóvenes que participaron en los proyectos tienen enormes desventajas a la hora de insertarse en el mundo del trabajo y en la sociedad en general.

Al finalizar los proyectos se puede identificar que algunos jóvenes (aunque no es suficiente) lograron mejorar su posicionamiento socioeducativo y su inclusión al mundo del trabajo. Los datos obtenidos así lo confirman como podemos constatar en el siguiente cuadro:

Deteniéndonos en una mirada estrictamente educativa, un técnico del PMO comentaba que se había logrado instalar una conciencia en los jóvenes la necesidad de educarse, que si bien no hay una relación directa entre finalizar la escuela y conseguir un trabajo, sí para los jóvenes se visualizaban mejores oportunidades.

El análisis de las entrevistas mostró que los jóvenes beneficiarios de los programas tuvieron pocas posibilidades de inclusión laboral, ya que poseen escasa o nula calificación para el ingreso al mercado formal de trabajo y por consiguiente el único acceso viable es a puestos de trabajos precarios, - - los que se caracterizan por bajos ingresos y transitoriedad - o bien, están sujetos al desempleo siendo la inestabi- 
lidad y la desprotección laboral condiciones muy frecuentes entre ellos. Estas formas de inclusión profundizan la ausencia de proyectos a futuro y la desvaloración del conocimiento y la educación como motores de ascenso social; por lo tanto, es necesario identificar de qué manera el tránsito por un proyecto modifica otras esferas de la vida personal y comunitaria de estos jóvenes.

Respecto de ello al inicio del proyecto los jóvenes fueron consultados respecto a sus sueños, algunas respuestas fueron las siguientes:

No sé. No tengo (varón, 19 años).

Me mudaría y me iría del barrio. Por el tema de la inseguridad (mujer, 21 años).

Salir alguna vez todos de la Villa, y yo me prendo en eso (mujer, 18 años).

Terminar la casa. Trabajaría para poder ayudar y terminarla más rápido (varón, 22 años).

Que no haya más pobreza en la villa. Que haya comedores de noche (mujer, 17 años).

Una vida mejor que la de ahora (varón, 20 años).

Ninguno. No pienso en eso. No da (varón, 18 años).

Puedo afirmar a partir de mi experiencia de campo, que a la crisis del mercado de trabajo se suma el hecho de que estos jóvenes no encuentran espacios de socialización habituales para jóvenes de otras clases sociales, como la escuela, el trabajo y el barrio. Esta situación se agudiza cuando las familias se encuentran desocupadas y en muchas ocasiones — dada las condiciones de existencia- se interrumpe el diálogo entre ellos respecto del futuro generándose situaciones de mayor vulnerabilidad. O sea, a los oídos sordos del Estado y la sociedad se le agregan los oídos sordos de la familia. Escuchar a los jóvenes acerca de su propia situación aporta elementos valiosos para comprender el impacto que tienen estos proyectos en su vida.

Ambos proyectos presentaron propuestas de formación para el trabajo; como señala Jacinto (2007) los puentes entre «educación y trabajo» se hacen cada vez más difíciles de concretar. Al iniciar el proyecto una de nuestras preocupaciones se refería al imaginario que tenían estos jóvenes beneficiarios de ambos planes, acerca de la educación en general y de la escuela en particular, para ello indagamos acerca de la importancia que le otorgaban a la escuela. Las respuestas más frecuentes era para aprender (57,1\%), para conseguir trabajo y para el futuro $(9,5 \%)$ y el $19 \%$ sostenía que para nada. Esta respuesta 
era coincidente en todas las situaciones aún en el caso de los jóvenes que habiendo abandonado la escuela buscaban trabajo y no encontraban. He aquí algunas de las respuestas de los jóvenes:

Sirve para tener tu casa, para dedicarte a algo, si no tenés trabajo no comes, no tenes casa, no existís (mujer, 18 años).

Para que no falte nada en la casa (Varón, 23 años).

Para una persona grande para mantener a sus hijos. Para una persona joven para mantener los estudios (varón, 21 años).

En general, los espacios de formación unieron la capacitación técnica con otras habilidades necesarias para enfrentar la realidad. Las organizaciones también trabajaron aspectos que tuviesen en cuenta el contexto en que se desarrollaban los jóvenes y las dificultades que enfrentaban.

Son chicos que se habían criado, digamos, de los noventa para acá, tenían en ese momento 18, 20 años y hacia 10,12, que los padres no tenían trabajo, se habían criado en ese contexto, sin escuela, de paro en paro, en el medio la reforma educativa también. Bueno entonces en ese momento, lo que se hizo fue trabajar desde ese lugar, desde la generación de expectativas nuevas, de ver que se les movía a los chicos internamente, con esto de conseguir trabajo, de la posibilidad de conseguir trabajo, qué significaba para ellos tener un sueldo o no tenerlo, ese tipo de cuestiones, de la cultura del trabajo (profesional mujer, 33 años).

Al finalizar su tránsito por los espacios de capacitación, el 53\% de los jóvenes describían una clara mejora en sus posibilidades de inclusión laboral, aunque tuvieran una inserción precaria en el mercado. Si bien los empleos a los que se incorporaron fueron de poca remuneración y no registrados, los consideraban como positivos, dado que creían que esa situación sería transitoria y una posibilidad económica para ayudar a su familia mientras decidían que hacer en su futuro.

El 38\% de los jóvenes presentaron trayectorias laborales caracterizadas por inserciones débiles, de alta rotación, tendientes a seguir reproduciéndose. La desocupación bastante continua precede a empleos temporarios que parecen ser, en la perspectiva de ciertos jóvenes, la única posibilidad de inserción laboral.

Entre los jóvenes que hicieron la capacitación y recibieron apoyo técnicos y financieros del Programa Manos a la Obra para la creación de emprendimientos productivos, hallamos que sólo el $11 \%$ de los jóvenes avanzaron con sus proyectos y cuentan hoy con alternativas 
concretas de trabajo que desarrollan ya sea en forma individual o asociativamente.

Entre los jóvenes que se encontraban inactivos; es decir, que no trabajan ni buscan trabajo, pero que quería terminar sus estudios secundarios antes de ingresar al mercado laboral, el 83\% eran adolescentes o jóvenes entre 17 y 20 años.

La participación en ambos proyectos parece haber dejado en todos los jóvenes (93\%) aprendizajes positivos vinculados con habilidades para la búsqueda de empleo; como por ejemplo, el saber desenvolverse en una entrevista laboral, organizar un currículum vitae o el vencer la timidez.

$\mathrm{Al}$ inicio de los programas, el 71,4\% de los jóvenes se sentía discriminado, especialmente por la escuela y los adultos. Los motivos señalados eran:

Piensan que todos somos criminales (varón, 18 años).

Creen que somos violentos o drogadictos (varón, 21 años)

No les gusta nuestra forma de vestirnos ni nuestro aspecto físico (mujer, 19 años).

La mayoría de los jóvenes experimentan un fuerte rechazo por lo que ellos identifican como «la gente con más dinero» y especialmente contra la policía y la seguridad de los boliches. Las razzias, las muertes de adolescentes en manos de policías, el trato que reciben al ser detenidos sin causa alguna, así lo confirman. En un grupo de discusión la mayoría llegaba a la conclusión de que cuando pisaban la tierra —en alusión a llegar a la Villa - se sentían tranquilos. El joven de estos sectores sufre, dado el contexto que le toca vivir, la falta de apoyo, referencia e ideales claros agravado esto por el efecto que produce en su constitución psíquica la pobreza estructural. La desocupación como marca de identidad y no como algo temporario, así como el sentimiento de vergüenza, hablan también de condiciones erosionantes en la construcción del sujeto.

\section{REFLEXIONES FINALES}

Como hemos puesto en evidencia en los capítulos precedentes, la educación, la formación y el empleo reduce a los jóvenes pobres a un círculo reproductor de pobreza. No logran trabajos dignos, porque no tienen formación y no obtienen formación porque necesitan obtener 
ingresos. A ello se agregan las crecientes segmentaciones culturales, entre las que se destacan las propias del mercado laboral, que se expresan en que ni los padres ni los abuelos de esos jóvenes se desempeñaron en el mercado de trabajo formal. El círculo se cierra con el pronóstico de que sus hijos tampoco lo lograrán. Hasta no hace muchos años, se aprendía a trabajar, trabajando. Los niños y jóvenes que no tenían como perspectiva futura el tránsito por sistemas educativos terciarios o universitarios, se unían a sus padres, abuelos, tíos, para el aprendizaje de ciertos oficios. Actualmente ya no hay trabajos decentes, ni siquiera relativamente estables, pero no sólo para ellos, sino tampoco los hubo en su familia por lo que no podemos dejar de preguntarnos ¿qué modelo tiene, cómo sabe y concibe qué es el trabajo un joven que nunca vio a nadie con un trabajo digno en su familia?

$\mathrm{Al}$ acceder por sí mismos a trabajos inestables y precarios, o a planes sociales de emergencia que exigen contraprestaciones que las más de las veces funcionan "como si fueran trabajo», perdieron la posibilidad no solo de aprender haciendo sino que también se perdió el trabajo como eje central en la constitución de su identidad social. Autores como Pérez Islas y Urteaga (2001) —entre otros - suelen decir que para los jóvenes el trabajo se desdibuja como eje de la organización personal aunque es preciso diferenciar situaciones socioeconómicas y capitales culturales y sociales, tramos etarios, etcétera, para comprender con mayor profundidad estos procesos.

Como indican las estadísticas, la educación resulta en la actualidad necesaria, pero no suficiente para acceder a un trabajo de calidad. A pesar de la mejora en el acceso a niveles de inclusión escolar son bajos para los sectores más empobrecidos de la sociedad; cuando los sujetos se incorporan a la escuela sin disponer de genuinos recursos; sin condiciones mínimas de infraestructura; sin posibilidades de promoción educativa y sin posibilidades de permanencia, todo esto se traduce en exclusión. Gentili (2001) se refiere a este fenómeno como «situación de exclusión de un sistema educativo incluyente».

Consideramos necesario resaltar que en el transcurso de la investigación lo que observamos es que el éxito de la política de empleo no necesariamente se vincula al logro de mejores condiciones en la inserción laboral. Las organizaciones y los jóvenes identifican fortalezas y ganancias a lo largo del proyecto, que pueden identificarse de la siguiente manera. 
a) Trabajo en red: la implementación de los proyectos planteó la articulación de distintos ámbitos en los cuales aparecían efectores del sistema educativo formal, del sistema de salud local, efectores socioeducativos no formales, organismos municipales y provinciales, etcétera. Y si bien cada uno de ellos tiene una configuración singular, se propusieron abordar en red las dificultades de integración social de los jóvenes beneficiarios de los programas y administrar los recursos sin sesgos clientelares.

b) Promoción del protagonismo juvenil: las acciones y la estrategia planteada se centró en la idea de que los jóvenes beneficiarios pudieran convertirse en gestores de acciones destinadas a promover sus propios intereses constituyéndose en actores sociales; ser capaces de desplegar potencialidades (orientadas desde la educación para el trabajo); desarrollar una mirada estratégica articulando su propio beneficio con el de su comunidad (ciudadanía política) y adquirir una mayor credibilidad, vinculación y referencialidad con las organizaciones comunitarias.

c) Identidad de grupo: como resultado de los dieciocho meses compartidos en los proyectos, los jóvenes provenientes de diferentes sectores de la Villa conformaron un grupo de permanencia y referencia estableciendo vínculos de compañerismo y amistad entre ellos. Sostenemos que, para su participación en las decisiones colectivas, los intereses particulares necesitan algo más que el reconocimiento de su libertad de expresión para ser tenidos en cuenta y es la oportunidad, la capacidad y la elección de influir con poder en las decisiones que los afectan. Los jóvenes se consideraron gestores de sus proyectos y eso fue su mejor ganancia.

Entre las semejanzas de ambos proyectos podemos señalar que se intentó realizar un abordaje diferente y más amplio de los problemas generales del colectivo juvenil, fundamentalmente, porque en la atención de estos temas se evidencia un mayor énfasis en la búsqueda de una integración de tipo social y cultural más cercana a la noción de construcción y ampliación de ciudadanía. Sin embargo, en relación con el tratamiento específico del problema del empleo en este grupo etario las soluciones propuestas para su mejoramiento reflejan continuidades tanto en las estrategias de intervención como en los lineamientos políticos que los sustentan.

Por su parte, la matriz básica en materia de intervención estatal que caracterizó a las políticas sociales compensatorias en la década 
pasada y a partir de las cuales se institucionalizaron las ideas fuerza provenientes de una raíz neoliberal - focalización, descentralización y privatización-, permanecen vigentes en ambas propuestas imposibilitando, en cierta medida, la aparición de perspectivas diferentes que promuevan la generación de alternativas más integrales y que contribuyan a un alejamiento efectivo de las situaciones de exclusión social. Los proyectos se convierten según Beck (1998), en una especie de «sala de espera» para los jóvenes desempleados, pero que no pueden cambiar la situación básica que es la falta de trabajo.

Por otro lado, si ponemos el énfasis en las posibilidades de inserción laboral, el panorama del trabajo para los jóvenes de Villa Itatí se muestra poco promisorio. Los mismos están acostumbrados a que el trabajo sea precario, inestable, mal remunerado y que el trato hacia ellos no siempre sea respetuoso. Los intentos por superar esta situación parten siempre de iniciativas individuales, como cambiar de trabajo cuando las condiciones son desfavorables, o intentar estudiar y capacitarse para dejar atrás los empleos precarios. En todo caso, los jóvenes perciben que una mejora definitiva de su situación se dará en el futuro y no será a través de un cambio en las reglas de juego que les tocó en suerte sino tratando de adaptarse a la situación que viven.

La trayectorias laborales de los jóvenes y la sensación permanente de que las tempranas experiencias de explotación y precariedad laboral no pasarán, configuran un panorama en el que aparentemente el trabajo no genera identidad colectiva y; por lo tanto, tampoco da cabida a la acción colectiva de reivindicación de derechos laborales. En todo caso, los jóvenes se identifican como colectivo sólo por ser jóvenes; es decir, en oposición a los adultos, lo cual no es poco decir.

La carencia de iniciativas de acción colectiva en defensa de los derechos laborales, por parte de los jóvenes, no sólo se explica porque éstos estén esperando que mejore su situación laboral en el futuro; el sólo hecho de que una gran proporción de los jóvenes trabaje en el sector informal explica la enorme dificultad de establecer una asociación de trabajadores. Ciertamente, a pesar del persistente deterioro de las condiciones laborales, no existen evidencias de que los jóvenes aspiren a una participación política a través de la organización laboral.

La Plata (ARgentina), AgOsto 2011

RECIBIDO: AGOSTO 2011

ACEPTADO: OCTUBRE 2011 


\section{REFERENCIAS BIBLIOGRÁFICAS}

AuYERO, J. et al. (1992): «Juventud: hábitos y fluctuaciones». Revista Nueva Sociedad $\mathrm{N}^{\circ} 117$. Caracas: Nueva Sociedad.

ABDALA, E. (2004): «Formación y empleabilidad de jóvenes en América Latina». En M. MOLPECERES PASTOR: Identidades y formación para el trabajo en los márgenes del sistema educativo: escenarios contradictorios en la garantía social. Montevideo: CINTERFOR.

Balardini, Sergio (2005): «Políticas locales de juventud en municipios argentinos». En Políticas locales de juventud. Colección PROSUR. Buenos Aires: Fundación Friedrich Ebert Stiftung.

BARBeito, A. y R. Lo Vuolo (1995): La pobreza... de la política contra la pobreza. Buenos Aires: Editorial Miño y Dávila.

BarbetTI, PABLo (2007): «Programas sociolaborales juveniles y desarrollo local. Análisis de una intervención en Gran Resistencia (Chaco) desde las opiniones y valoraciones de los actores locales». Revista IIED-AL No66. Buenos Aires: Instituto Internacional de Medio Ambiente y Desarrollo.

Bendit, René (2009): Transiciones a la vida adulta. Principales dimensiones analíticas. Buenos Aires: FLACSO.

CACHÓN RODRÍGUEZ, L. (1989): «Inserción laboral de jóvenes en la Unión Europea». Papeles de la Oficina Técnica No4. Montevideo: CINTERFOR.

CASAL, JOAQUIM (2006): «Aportaciones teóricas y metodológicas a las sociología de la juventud desde la perspectiva de la transición». Papers №79. Barcelona: Universidad Autónoma de Barcelona.

CORTAZzO, I. y L. FARÍAS (2007): «Construyendo estrategias de inclusión laboral con los jóvenes». En Patricia SChettini y Cecilia Senen GONZÁLEZ: Trabajo y relaciones laborales: sector formal e informal. Buenos Aires: Prometeo y Universidad Nacional de La Matanza.

DÁVILA, ÓsCAR; FELIPE GHIARDO y CARLOS MEDRANO (2006): Los desheredados. Trayectorias de vida y nuevas condiciones juveniles. Valparaíso: Ediciones CIDPA.

, RAÚl IRRAZABAL y Astrid OYARzÚn (1995): «Los jóvenes como comunidades realizadoras: entre lo cotidiano y lo estratégico». En $\mathrm{Ni}$ adaptados ni desadaptados sólo jóvenes. Siete propuestas de desarrollo juvenil. Santiago: PIIE.

DANANI, ClAUDIA (2003): Entre el trabajo y la política. Las reformas de las políticas sociales argentinas en perspectiva comparada. Buenos Aires: Biblos.

DiNAJU (2008): Informe de la situación laboral y educativa de los jóvenes en Argentina. Buenos Aires: Dirección General de Juventud.

Duschastzky, S y C. COREA (2002): Chicos en banda, los caminos de la subjetividad en el declive de las instituciones. Buenos Aires: Paidós. 
FARÍAS, MARÍA LOURDES (2004): «De la fragmentación a la organización. El desafío de construir opciones educativas para jóvenes en situación de pobreza». Conferencia Internacional de Sociología de la Educación. Buenos Aires.

KRAUSKOPF, DinA (2004): Perspectiva sobre la condición juvenil y su inclusión en las políticas públicas. CLACSO.

LEPORE-SCHLESER (2007): «El desempleo juvenil en Argentina: perfiles y dinámicas». $7^{\circ}$ Congreso Nacional de Estudios del Trabajo. Buenos Aires.

LiNDEMBOIM, J. (1998): Los problemas del empleo a fines del siglo XX. Buenos Aires: Siglo XXI Editores.

—, L. SERINO y M. GONZÁLEZ (2000): «La precariedad como forma de exclusión.» Cuadernos del CEPED No 4. Buenos Aires: IIE/FCE/UBA.

LO VuOLO, R. y A. BARBEITO (1994): La nueva oscuridad de la política social. Del Estado Populista al Neoconservador. Buenos Aires: Niño y Dávila Editores.

PÉREZ ISLAS, JosÉ ANTONIO y MARITZA URTEADA (2001): «Los nuevos guerreros del mercado. Trayectorias laborales de jóvenes buscadores de empleo». En ENRIQUE PIECK (editor): Los jóvenes y el trabajo: la educación frente a la exclusión social. México: IMJ.

REDONDO, JESÚs (2000): «La condición juvenil: entre la educación y el empleo». Última Década $\mathrm{N}^{\circ} 12$. Valparaíso: Ediciones CIDPA.

Ríos Segovia, PAtricio (2008): «El habitus de la edad». Última Década $\mathrm{N}^{\circ} 28$. Valparaíso: Ediciones CIDPA.

RODRÍGUEZ, ERNESTO (1994): «Propuesta de plan integral de juventud: políticas de juventud y estrategias de desarrollo en la antesala del año 2000». Montevideo: Instituto Nacional de la Juventud.

SÁEZ Marín, Juan (1988): El frente de juventudes. Política de juventud en la España de la posguerra (1937-1960). Madrid: Siglo XXI Editores.

SAlviA, A. (2008) «Introducción: la cuestión juvenil bajo sospecha». En A. Salvia (compilador): Jóvenes promesas. Trabajo, educación y exclusión social de jóvenes pobres en la Argentina. Buenos Aires: Editorial Miño y Dávila.

WELLER, J. (2003): «La problemática inserción laboral de los y las jóvenes». Serie Macroeconomía del Desarrollo No28. Santiago: CEPAL. 